\title{
Quantum Strips on Surfaces *
}

\author{
David Krejčiř́ík ${ }^{\dagger}$ \\ Laboratoire de Mathématiques, Université de Reims, \\ Moulin de la Housse, BP 1039, 51687 Reims cedex 2, France \\ E-mail: david.krejcirik@univ-reims.fr
}

\begin{abstract}
Motivated by the theory of quantum waveguides, we investigate the spectrum of the Laplacian, subject to Dirichlet boundary conditions, in a curved strip of constant width that is defined as a tubular neighbourhood of an infinite curve in a two-dimensional Riemannian manifold. Under the assumption that the strip is asymptotically straight in a suitable sense, we localise the essential spectrum and find sufficient conditions which guarantee the existence of geometrically induced bound states. In particular, the discrete spectrum exists for non-negatively curved strips which are studied in detail. The general results are used to recover and revisit the known facts about quantum strips in the plane. As an example of non-positively curved quantum strips, we consider strips on ruled surfaces.
\end{abstract}

\section{Introduction}

The theory of quantum waveguides constitutes a beautiful domain of mathematical physics in which one meets an interesting interaction of analysis and geometry. Recall that the configuration space $\Omega$ of a waveguide is usually modelled by tubular neighbourhoods of infinite curves in $\mathbb{R}^{d}, d=2,3$ (quantum strips, tubes), or surfaces in $\mathbb{R}^{3}$ (quantum layers), while the dynamics is governed by the Laplace operator with Dirichlet boundary conditions. It is due to an admirable progress of mesoscopis physics that such models do really represent actual nanostructures which are produced in the laboratory nowadays. We refer to [6, 22] for the physical background and references.

A common, particularly interesting property of these systems is that the curvature of the reference curve or surface may produce bound states of the Laplacian below the essential spectrum. This phenomenon was demonstrated first in

*PREPRINT

†On leave of absence from

Nuclear Physics Institute, Academy of Sciences, 25068 Řež near Prague, Czech Republic

Date: April 25, 2002.

Key Words: quantum waveguides, quantum strips, Laplacian, Dirichlet conditions, bound states, Fermi coordinates, curvature, ruled surfaces, flat, geodesic, asymptotically geodesic. MSC2000: 58J50, 81Q10. 
a rigourous way by P. Exner and P. Šeba for curved strips in the plane, [10]. Numerous subsequent studies improved their result and generalised it to space tubes. For more information and other spectral and scattering properties, see the review paper [6] and references therein. The evidently more complicated case of quantum layers was investigated quite recently in [7, 8, 9].

Up to this time, the ambient manifold of the quantum waveguide has been usually identified with a flat Euclidean space $\mathbb{R}^{d}, d=2,3$. This restriction is obviously due to the physical reasons, however, at least from the mathematical point of view, one may be interested equally in the situations when it is a general Riemannian manifold $\mathcal{A}$ of dimension $d \geq 2$. The principal interest of the present work is to initiate this study by considering the simplest non-trivial case, $d=2$, when the configuration space $\Omega$ is a tubular neighbourhood of constant radius $a>0$ about an infinite curve $\Sigma$ on a surface $\mathcal{A}$.

Let us describe the contents of the paper. The strip configuration space $\Omega$ itself is properly defined in Section 2.1. Through all the paper, we suppose that the strip is globally parameterised by a system of geodesic coordinates based on the reference curve $\Sigma$. In accordance with [15], we call them Fermi coordinates, [11], although they had already been considered by C. F. Gauss. A comprehensive discussion of such a coordinate system has been given by F. Fiala, [12], in order to prove some isoperimetric inequalities; see also [17]. A modern definition of Fermi coordinates of tubes about a submanifold of a general Riemannian manifold can be found in 15]. We introduce them for our purposes in Section 2.2.

In Section 2.3, the Hamiltonian $H$ of our system is identified with the Friedrichs extension of the Laplacian, $-\Delta$ on $L^{2}(\Omega)$, which is expressed in Fermi coordinates and defined initially on $C_{0}^{\infty}(\Omega)$. The construction is based on the quadratic-form approach of [4, Chap. 6]. Two trivial classes of quantum strips are then mentioned in Section 2.4. If the curvature of the ambient space is identically equal to zero on $\Omega$, the strip is called flat and the spectrum of $H$ coincides with the spectrum of strips in the plane, [6]. A generalisation of straight strips in the plane is represented by geodesic strips, for which the reference curve is in addition a geodesic. In that case, we find that the spectrum is the interval $\left[\kappa_{1}^{2}, \infty\right)$, where $\kappa_{1}:=\pi /(2 a)$.

Section 3 is devoted to a heuristic analysis of of the Hamiltonian $H$. Using a unitary transformation, it can be identified with a Schrödinger-like operator with a potential expressed by means of the metric of $\Omega$. The latter operator acquires a very instructive form in the formal limit when the width of the strip tends to zero. In particular, we reveal an effective potential which is given by a combination of curvatures of $\Sigma$ and $\mathcal{A}$. The result is compared with the case of strips in the plane and used as a motivation for the spectral analysis of $H$ in the subsequent sections.

In Section 1 , we localise the essential spectrum under the assumption that the strip is asymptotically geodesic in a suitable sense. Using a Neumann bracketing argument together with the minimax principle, we find in Theorem 11 that the threshold of $\sigma_{\text {ess }}(H)$ is then bounded from below by $\kappa_{1}^{2}$. 
Section 5 is devoted to the analysis of the spectrum below the energy $\kappa_{1}^{2}$. Using a variational technique standard in the theory of quantum waveguides, we find two sufficient conditions which guarantee that this part of spectrum is not empty, $c f$ Theorems and 3 . These conditions require that the strip is nonnegatively curved in an integral sense; see (21) for the precise meaning of the statement. Combining these results with Theorem 1, we arrive at Corollary 11 which contains the main result of this paper concerning the existence of a nontrivial discrete spectrum in quantum strips.

Since the condition (21) is clearly satisfied for non-negatively curved strips, this situation is investigated in detail in Section 6. We simplify some assumptions, we have put on the geometry of $\Omega$, and sum up the spectral results in Theorem 1. Apart from a significant generalisation, it recovers and revisits the known results for the quantum strips in the plane.

To the best of our knowledge, it is for the first time when the spectrum of a curved strip embedded in a non-trivial manifold has been investigated. An exception is the paper [2], where I. J. Clark and A. J. Bracken deal with a special class of quantum strips in $\mathbb{R}^{3}$, which are made up from segments perpendicular to an infinite space curve $\Sigma$. They introduce the Hamiltonian in a formal way, derive the effective potential mentioned above and make some conjectures on the influence of the torsion of $\Sigma$ on the spectrum, however, do not perform any spectral analysis itself. Actually, their paper is a preliminary to [1], where bound states in space quantum waveguides with torsion are investigated. The strip of [2] is a part of a ruled surface $\mathcal{A}$ based on $\Sigma$; we examine this situation briefly in Section 7 .

We conclude the paper by Section 8, where some open problems and directions of a future research are mentioned. A particularly interesting question concerns possible applications to physics.

\section{Preliminaries}

\subsection{Definitions}

Let $\mathcal{A}$ be a non-compact two-dimensional Riemannian manifold of class $C^{2}$ and let $K$ denote its Gauss curvature. We require that $K$ is a continuous function on $\mathcal{A}$, which holds if $\mathcal{A}$ is of class $C^{3}$ or if it is embedded in $\mathbb{R}^{3}$. Even if it is not necessary for our construction, we shall assume that $\mathcal{A}$ is geodesically complete.

Let $\Sigma$ be a simple, infinite curve of class $C^{2}$ embedded in $\mathcal{A}$ and let $k$ denote its curvature. (We do not require that $\mathcal{A}$ is embedded in $\mathbb{R}^{3}$, however, if it is that case, $k$ means the geodesic curvature of $\Sigma$.) We may assume that $\Sigma$ is given by the image of the mapping $p: \mathbb{R} \rightarrow \mathcal{A}$ such that $\left|p^{\prime}\right|=1$. It represents the $C^{2}$-parameterisation of the curve by its arc length. We note that $k$ is a continuous function on $\Sigma$.

Let $a>0$ and $I:=(-a, a)$. The strip $\Omega$ of width $2 a$ is defined as the $a$-tubular neighbourhood of $\Sigma$ in $\mathcal{A}$ :

$$
\Omega:=\{x \in \mathcal{A} \mid \operatorname{dist}(x, \Sigma)<a\} .
$$


As usual, the distance dist $(x, \Sigma)$ means here the length of the minimal geodesic joining $x$ with $\Sigma$. We want to introduce the Laplacian in $\Omega$ and investigate its spectrum. Our strategy is to map the curved strip (1) onto the straight one, $\Omega_{0}:=\mathbb{R} \times I$, by the use of Fermi coordinates which are defined in the following subsection.

\section{$2.2 \quad$ Fermi Coordinates}

We denote by $T_{x} \mathcal{A}$ the tangent space to $\mathcal{A}$ at $x \in \mathcal{A}$ and recall that the exponential map, $\exp _{x}: T_{x} \mathcal{A} \rightarrow \mathcal{A}$, is the identification $t \mapsto \gamma_{t}(1)$, where $\gamma_{t}$ is the unique geodesic (parameterised by arc length) in $\mathcal{A}$ with $\gamma_{t}(0)=x$ and $\gamma_{t}^{\prime}(0)=t$. We define

$$
\mathcal{L}: \mathbb{R}^{2} \rightarrow \mathcal{A}:\left\{(s, u) \mapsto \exp _{p(s)}(u n(s)) \mid n \in N_{p} \Sigma\right\},
$$

where $N_{p} \Sigma$ denote the orthogonal complement of $T_{p} \Sigma$ in $T_{p} \mathcal{A}$, and always assume that

$$
\mathcal{L}: \Omega_{0} \rightarrow \Omega \text { is a diffeomorphism for some } a>0 .
$$

Then the inverse of $\mathcal{L}$ determines the system of Fermi "coordinates" $(s, u)$ and one has

$$
\Omega=\mathcal{L}\left(\Omega_{0}\right) .
$$

Remark 1. Hereafter we shall use the standard component notation of tensor analysis with the range of indices being 1,2 and associate them with Fermi coordinates via the identification, $(1,2) \leftrightarrow(s, u)$. The partial derivatives will be denoted by commas. From now on the curvature $K$ shall be considered as a function of Fermi coordinates $(s, u) ; k$ is a function of $s$.

The metric tensor of $\Omega$ in Fermi coordinates is given by $G_{i j}:=\left\langle\mathcal{L}_{, i}, \mathcal{L}_{, j}\right\rangle$, where " $\langle\cdot, \cdot\rangle$ " denotes the inner product induced by the Riemannian metric on $\mathcal{A}$. Note that $s \mapsto \mathcal{L}(s, u)$ traces the curves parallel to $\Sigma$ at a fixed distance $|u|$ and that the curve $u \mapsto \mathcal{L}(s, u)$ is a unit-speed geodesic orthogonal to $\Sigma$ for any fixed $s$. The generalised Gauss Lemma, [15. Sec. 2.4], implies that these curves meet orthogonally and one arrives at the diagonal form of the metric tensor

$$
\left(G_{i j}\right)=\left(\begin{array}{cc}
f(s, u)^{2} & 0 \\
0 & 1
\end{array}\right) .
$$

According to 16, 18, the function $f$ is continuous and has continuous partial derivatives $f_{, u}, f_{, u u}$ satisfying the Jacobi equation

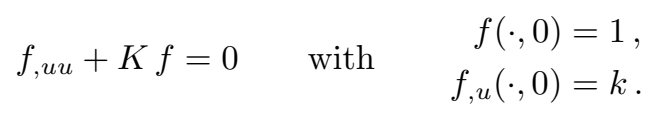

The determinant of the metric tensor, $G:=\operatorname{det}\left(G_{i j}\right)=f^{2}$, defines through $d \Omega:=G(s, u)^{\frac{1}{2}} d s d u$ the surface element of the strip. 
Remark 2. If $\Sigma$ was a compact curve, then the condition $\langle\mathrm{H} 1\rangle$ could always be achieved for sufficiently small $a$. Recall also that the same holds true for infinite strips in the plane if one assumes in addition that $\Omega$ does not overlap, [6]. In our case, the situation is analogous. The inverse function theorem implies that $\mathcal{L}: \Omega_{0} \rightarrow \Omega$ is a local diffeomorphism provided $f$ is uniformly strictly positive and bounded, $c f$ posterior assumption $\langle\mathrm{H} 2\rangle$. This can be achieved for $a$ small enough because $f(\cdot, 0)=1$. The condition $\langle\mathrm{H} 1\rangle$ will be then fulfilled globally if we do not allow in addition an overlapping of the strip.

We needed the ambient manifold $\mathcal{A}$ just in order to define the strip by means of (1) or (3). Once the construction is over, we may forget about the rest of $\mathcal{A}$ and consider its part $\Omega$ only. It will be our configuration space. Note that the closer $\bar{\Omega}$ is a manifold with boundary.

\subsection{Hamiltonian}

After geometric preliminaries, let us define the Hamiltonian of our system. We consider a non-relativistic quantum particle within the two-dimensional region $\Omega$ of impenetrable boundary. As usual, we put $\hbar^{2} /(2 m)=1$, where $\hbar$ denotes Planck's constant and $m$ the mass of the particle. Then the Hamiltonian could be identified with the Laplace operator, $-\Delta$ on $L^{2}(\Omega)$, with an appropriate domain of functions which vanish on $\partial \Omega$. However, we proceed differently and always understand this Laplacian in the generalised (form) sense.

In detail, using Fermi coordinates, we shall identify the Hilbert space $L^{2}(\Omega)$ with $\mathcal{H}:=L^{2}\left(\Omega_{0}, d \Omega\right)$. Let us consider the quadratic form on $\mathcal{H}$ given by

$$
Q(\psi, \phi):=\left(\psi_{, i}, G^{i j} \phi_{, j}\right)_{\mathcal{H}}, \quad \operatorname{Dom} Q:=W_{0}^{1,2}\left(\Omega_{0}, d \Omega\right),
$$

where $\left(G^{i j}\right)$ is the inverse of $\left(G_{i j}\right)$. Assuming that the metric is uniformly elliptic in the sense that the condition

$$
\exists c_{ \pm}>0 \quad \forall(s, u) \in \Omega_{0}: \quad c_{-} \leq f(s, u) \leq c_{+}
$$

is valid, it follows that the form $Q$ is non-negative and closed on its domain. Consequently, there exists a non-negative self-adjoint operator $H$ associated to $Q$ which satisfies $\operatorname{Dom} H \subset \operatorname{Dom} Q$. It will be our Hamiltonian. We refer to [4, Chap. 6] for more details and proofs concerning the above construction.

Remark 3. Although $H$ is formally equal to the operator $-G^{-\frac{1}{2}} \partial_{i} G^{\frac{1}{2}} G^{i j} \partial_{j}$, ie the Laplacian, $-\Delta$, expressed in Fermi coordinates, we shall be particularly concerned not to assume that the metric is differentiable. If, however, the metric is sufficiently smooth then the operator $H$ is indeed given by this expression with Dirichlet boundary conditions in the classical sense. We stress that under our assumptions, $f$ is only continuous w.r.t. $s$. 


\subsection{Flat and Geodesic Strips}

Assume that the strip is flat in the sense that the curvature $K$ is equal to zero everywhere on $\Omega, K \equiv 0$. Then the Jacobi equation (5) has the exact solution

$$
f(s, u)=1+u k(s) .
$$

This is a well-known result for the strips in the plane, however, we note that the same holds as well for the strips on cylinders, on surfaces of the shape of a corrugated iron, etc. Since the Hamiltonian is expressed via the metric which depends on $f$ only, we may immediately adapt to the flat strips all the results which has been previously derived for the quantum strips in the plane, [6]. In particular, the discrete spectrum will always exist as soon as the strip is nontrivially curved, $k \not \equiv 0$, and asymptotically straight, $k \stackrel{\infty}{\longrightarrow} 0$.

On the contrary, if (in addition to $K \equiv 0$ ) the reference curve is a geodesic, $k \equiv 0$, then the function $f$ equals 1 identically, and therefore

$$
H=H_{0}:=-\Delta_{D}^{\Omega_{0}} \quad \text { on } \quad L^{2}\left(\Omega_{0}\right) .
$$

Consequently, the discrete spectrum is empty and

$$
\sigma\left(H_{0}\right)=\sigma_{\mathrm{ess}}\left(H_{0}\right)=\left[\kappa_{1}^{2}, \infty\right),
$$

where $\kappa_{1}^{2}$ denotes the first eigenvalue of the Dirichlet Laplacian on the transverse section, $-\Delta_{D}^{I}$. These systems generalises the straight strips in the plane and will be called here geodesic. We will use them as a comparative class of quantum strips whose spectrum is known explicitly.

The operator $-\Delta_{D}^{I}$ occurs often in the present work. We note that it is nothing else than the quantum Hamiltonian of the one-dimensional infinite square well of width $2 a$. In what follows we shall use its family of eigenfunctions $\left\{\chi_{n}\right\}_{n=1}^{\infty}$ which is given by

$$
\chi_{n}(u):= \begin{cases}\sqrt{\frac{1}{a}} \cos \kappa_{n} u & \text { if } n \text { is odd, } \\ \sqrt{\frac{1}{a}} \sin \kappa_{n} u & \text { if } n \text { is even, }\end{cases}
$$

where $\kappa_{n}^{2}:=\left(\kappa_{1} n\right)^{2}$ with $\kappa_{1}:=\pi /(2 a)$ are the corresponding eigenvalues. The ground-state $\chi_{1}$ will be very important for us because it represents a generalised eigenvector of the geodesic strip corresponding to the threshold of the essential spectrum (8).

\section{Motivation}

This part is devoted to heuristic considerations in order to motivate the spectral analysis of the Hamiltonian in the following sections. It is possible, but beyond the scope of this paper, to examine the conditions under which the thin-width limit process below is justified. Since we use it just for motivation purposes, 
we shall do the limit in a formal way only. To this end (but only through this section!), we shall assume that $f$ is an analytic function.

Let us recall first the observation which initiated the attempts to prove the existence of bound states in quantum strips in the plane, $c f$ 19, 21, 3, 26. The Hamiltonian of such a strip is unitarily equivalent to a Schrödinger-like operator with a potential expressed by means of the curvature $k$ of the reference curve and the transverse coordinate $u \in I$. Making formally the thin-width limit, $a \rightarrow 0$, in the expression for the the transformed Hamiltonian, the potential becomes equal to $-k^{2} / 4$. The latter always represents an attractive interaction as soon as the strip is non-trivially curved, $k \not \equiv 0$, and asymptotically straight, $k \stackrel{\infty}{\longrightarrow} 0$. Consequently, the limit operator possesses bound states below its essential spectrum. As we have mentioned in the introduction, one proves that these bound states "survive" also in the actual quantum strips of non-zero widths.

In order to find the effective potential in our situation, we introduce the unitary transformation $U: \mathcal{H} \rightarrow L^{2}\left(\Omega_{0}\right)$ given by $\psi \mapsto G^{\frac{1}{4}} \psi$, which leads to

$$
\tilde{H}:=U H U^{-1}=-G^{-\frac{1}{4}} \partial_{i} G^{\frac{1}{2}} G^{i j} \partial_{j} G^{-\frac{1}{4}} .
$$

Commuting $G^{-\frac{1}{4}}$ with the gradient components, we cast this operator into a form which has a simpler kinetic part but contains a potential,

$$
\tilde{H}=-\partial_{i} G^{i j} \partial_{j}+V \quad \text { with } \quad V:=\left(G^{i j} J_{, j}\right)_{, i}+J_{, i} G^{i j} J_{, j},
$$

where $J:=\ln G^{\frac{1}{4}}$. This expression is valid for any smooth metric $G_{i j}$. Employing the particular form (4) of our metric tensor together with the Jacobi equation (5), we get

$$
V=\frac{1}{f^{2}}\left[\frac{1}{2} \frac{f_{, s s}}{f}-\frac{5}{4}\left(\frac{f_{, s}}{f}\right)^{2}\right]-\frac{1}{2} K-\frac{1}{4}\left(\frac{f_{, u}}{f}\right)^{2} .
$$

To make the limit when the width of the strip, $2 a$, tends to zero, we note first that the function $f$ admits, as a solution of (5), the following asymptotic expansion w.r.t. $u$ :

$$
f(s, u)=1+u k(s)-\frac{1}{2} u^{2} K(s, 0)+r(s, u),
$$

where the remainder $r$ is $\mathcal{O}\left(u^{3}\right)$ for any fixed $s$. Putting this expansion into (12) and (11), and making the limit $u \rightarrow 0$ in the expression for $V$ and $G^{i j}$, we see that, up to higher order terms in $u \in I$, the operator $\tilde{H}$ decouples formally into the direct sum of the operators

$$
-\Delta^{\mathbb{R}}+V_{\text {eff }} \quad \text { on } \quad L^{2}(\mathbb{R}) \quad \text { and } \quad-\Delta_{D}^{I} \quad \text { on } \quad L^{2}(I),
$$

where

$$
V_{\text {eff }}(s):=-\frac{1}{4} k(s)^{2}-\frac{1}{2} K(s, 0) .
$$


The first term in $V_{\text {eff }}$ is identical with the effective potential for the thin strips in the plane, while the second one reflects the fact that our strip is in addition embedded in a curved manifold now.

Assume that the curvatures $k$ and $K$ vanish at the infinity of the strip. In distinction to the planar case, the potential (15) may not represent an attractive interaction for any non-trivially curved strip. (For, it suffices to consider the strip constructed over a geodesic, $k \equiv 0$, on a surface of negative curvature.) Nevertheless, if the curvature $K$ is, say, non-negative (and $k \not \equiv 0$ provided $K \equiv 0$ ), then the potential $V_{\text {eff }}$ always represents an attractive interaction. Consequently, the direct sum of the limit operators of (14) possesses bound states below its essential spectrum. The aim of this paper is to state an analogous sufficient condition which guarantees the existence of a non-trivial discrete spectrum for the actual Hamiltonian $H$ of the strips of non-zero widths.

To conclude this section, we stress again that the thin-width-limit procedure we have used to derive the operators (14) and the effective potential (15) of thin strips is formal only. (One reason is that the transverse operator $-\Delta_{D}^{I}$ gives rise to infinite normal oscillations as $a \rightarrow 0$.) Nevertheless, we note that a similar thin-neighbourhood limit was performed rigourously by R. Froese and I. Herbst, [13], in efforts to treat the time evolution around a compact $n$ dimensional submanifold of $\mathbb{R}^{n+m}, m \geq 1$. There the confinement was realised by a harmonic potential transverse to the manifold and the limit was carried out by means of a dilation procedure followed by averaging in the normal direction. The situation when $\mathbb{R}^{n+m}$ is replaced by a Riemannian manifold of the same dimension was treated formally in [23]; there one can also recover the effective potential (15).

\section{Essential Spectrum}

In Section 2.4, we have seen that the essential spectrum of a geodesic strip $(k, K \equiv 0)$ starts by the first eigenvalue $\kappa_{1}^{2}$ of the transverse operator $-\Delta_{D}^{I}$. Since the metric tensor is the identity matrix $(f \equiv 1)$ in this case and the essential spectrum is determined by the behaviour of the metric at infinity only, we expect that the same will hold true if a curved quantum strip behaves like a geodesic strip asymptotically in the sense

$$
f \stackrel{\infty}{\longrightarrow} 1 \text {. }
$$

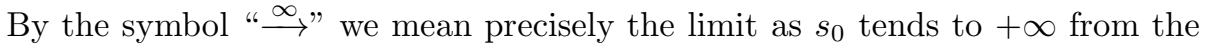
supremum over $(s, u) \in \Omega_{0} \backslash \Omega_{0, \text { int }}$, where $\Omega_{0, \text { int }}:=\left(-s_{0}, s_{0}\right) \times I$.

Remark 4. Note that the assumption $\langle\mathrm{H} 3\rangle$ together with $\langle\mathrm{H} 1\rangle$ implies the condition $\langle\mathrm{H} 2\rangle$ for any half-width less than $a$. In detail, since $f$ is continuous it is bounded locally, and cannot be equal to 0 on $\Omega_{0}$ because of $\langle\mathrm{H} 1\rangle$. The asymptotic assumption $\langle\mathrm{H} 3\rangle$ then controls the uniform behaviour of $f$ at infinity. 
Theorem 1. Assume $\langle\mathrm{H} 1\rangle,\langle\mathrm{H} 2\rangle$, and suppose that the strip is asymptotically geodesic, $\langle\mathrm{H} 3\rangle$. Then

$$
\inf \sigma_{\mathrm{ess}}(H) \geq \kappa_{1}^{2} .
$$

Proof: The idea is inspired with the proof of Theorem 4.1 in $[8]$. Let $s_{0}>0$, be $\Omega_{0 \text {,int }}$ as above and define $\Omega_{0, \text { ext }}:=\Omega_{0} \backslash \Omega_{0, \text { int }}$. The images of $\Omega_{0 \text {,int }}$ and $\Omega_{0 \text {,ext }}$ by the mapping $\mathcal{L}$ divide the strip $\Omega$ into an interior and exterior part, respectively. Imposing the Neumann boundary condition at the common boundary of the two parts, $s=s_{0}$, we arrive at the decoupled Hamiltonian $H^{N}=H_{\text {int }}^{N} \oplus H_{\mathrm{ext}}^{N}$. More precisely, it is obtained as the operator associated with the quadratic form $Q^{N}$ acting as (5), however, with the domain $\operatorname{Dom} Q^{N}:=\operatorname{Dom} Q_{\mathrm{int}}^{N} \oplus \operatorname{Dom} Q_{\mathrm{ext}}^{N}$, where

$$
\operatorname{Dom} Q_{\omega}^{N}:=\left\{\psi \in W^{1,2}\left(\Omega_{0, \omega}, d \Omega\right) \mid \psi(\cdot, \pm a)=0\right\}, \quad \omega \in\{\text { int, ext }\} .
$$

The corresponding quadratic forms $Q_{\omega}^{N}$ act like $Q$, however, on appropriately restricted Hilbert spaces $\mathcal{H}_{\omega}:=L^{2}\left(\Omega_{0, \omega}, d \Omega\right)$. Since $H \geq H^{N}$ and the spectrum of $H_{\mathrm{int}}^{N}$ is purely discrete, [4, Chap. 7], the minimax principle, [24, Sec. XIII. 1], gives the estimate

$$
\inf \sigma_{\text {ess }}(H) \geq \inf \sigma_{\text {ess }}\left(H_{\text {ext }}^{N}\right) \geq \inf \sigma\left(H_{\text {ext }}^{N}\right) .
$$

Hence it is sufficient to find a lower bound on $H_{\text {ext }}^{N}$. However, by virtue of (6) and (14), we have for all $\psi \in \operatorname{Dom} Q_{\mathrm{ext}}^{N}$ :

$$
\begin{aligned}
Q_{\text {ext }}^{N}[\psi] & \geq\left\|\psi_{, u}\right\|_{\mathcal{H}_{\text {ext }}}^{2} \geq\left(\inf _{\Omega_{0, \mathrm{ext}}} G^{\frac{1}{2}}\right)\left\|\psi_{, u}\right\|_{L^{2}\left(\Omega_{0, \mathrm{ext}}\right)}^{2} \\
& \geq\left(\inf _{\Omega_{0, \mathrm{ext}}}^{\frac{1}{2}}\right) \kappa_{1}^{2}\|\psi\|_{L^{2}\left(\Omega_{0, \mathrm{ext}}\right)}^{2} \geq\left(\inf _{\Omega_{0, \mathrm{ext}}} G^{\frac{1}{2}}\right)\left(\sup _{\Omega_{0, \mathrm{ext}}} G^{\frac{1}{2}}\right)^{-1} \kappa_{1}^{2}\|\psi\|_{\mathcal{H}_{\text {ext }}}^{2} .
\end{aligned}
$$

In the third inequality, we have used the bound $-\Delta_{D}^{I} \geq \kappa_{1}^{2}$. The obtained estimate on $Q_{\text {ext }}^{N}$ is valid for any metric of the block form (何) even if the function $f$ is replaced by a matrix. However, here we have $G^{\frac{1}{2}}=f$ and the infimum and supremum tend to 1 as $s_{0} \rightarrow \infty$ by the assumption $\langle\mathrm{H} 3\rangle$. The claim then easily follows by the fact that $s_{0}$ can be chosen arbitrarily large.

Remark 5. This threshold estimate is sufficient for the subsequent investigation of the discrete spectrum which is our goal in this paper. In order to prove the opposite estimate, one may employ a Dirichlet bracketing argument instead of the Neumann one we have used. Next, to show that all energies above $\kappa_{1}^{2}$ belong to the spectrum, one has to construct an appropriate Weyl sequence. This can be done under an assumption stronger than $\langle\mathrm{H} 3\rangle$ which involves derivatives of $f$ as well.

\section{Discrete Spectrum}

The aim of this section is to prove two conditions sufficient for the Hamiltonian to have a non-empty spectrum below $\kappa_{1}^{2}$. Since we have shown that 
the essential spectrum does not start below this value for the asymptotically geodesic strips, the conditions yields immediately the existence of curvatureinduced bound states. The proofs here are based on the variational idea of finding a trial function $\psi$ from the form domain of $H$ such that

$$
\tilde{Q}[\psi]:=Q[\psi]-\kappa_{1}^{2}\|\psi\|_{\mathcal{H}}^{2}<0 .
$$

The idea which goes back to J. Goldstone and R. L. Jaffe, [14], is to construct a trial function by deforming $\chi_{1}$ of (9), which represents a generalised eigenfunction of energy $\kappa_{1}^{2}$ for the geodesic strip. In particular, if the strip is geodesic, then $\tilde{Q}\left[\chi_{1}\right]=0$. The latter has to be understood in a generalised sense because $\chi_{1}$ is not integrable w.r.t. $s$ and as such it does not belong to $\operatorname{Dom} Q$. Let us use this function in the curved case. We start with a formal calculation:

$$
\begin{aligned}
\tilde{Q}\left[\chi_{1}\right] & =\left(\chi_{1, s}, f^{-1} \chi_{1, s}\right)+\left(\chi_{1, u}, f \chi_{1, u}\right)-\kappa_{1}^{2}\left(\chi_{1}, f \chi_{1}\right) \\
& =-\left(\chi_{1}, f_{, u} \chi_{1, u}\right)=\frac{1}{2}\left(\chi_{1}, f, \text {,u } \chi_{1}\right)=-\frac{1}{2}\left(\chi_{1}, K f \chi_{1}\right)
\end{aligned}
$$

where the inner product is in the Hilbert space $L^{2}\left(\Omega_{0}\right)$. The first equality is the definition of $Q$ and $\|\cdot\|_{\mathcal{H}}$, in the second one we have used the fact that $\chi_{1}$ does not depend on $s$ and integrated by parts w.r.t. $u$, in the third one we have integrated by parts once more, and the last equality follows by (5). The resulting integral will be well defined if we assume

$$
K \in L^{1}\left(\Omega_{0}, d \Omega\right) .
$$

Hence we obtain immediately

Theorem 2. Assume $\langle\mathrm{H} 1\rangle,\langle\mathrm{H} 2\rangle,\langle\mathrm{H} 4\rangle$, and suppose that

$$
\left(\chi_{1}, K \chi_{1}\right)_{\mathcal{H}}>0 \text {. }
$$

Then

$$
\inf \sigma(H)<\kappa_{1}^{2} .
$$

PROOF: It remains to regularise $\chi_{1}$ in such a way that the formal result (17) would be justified in a limit. For any $n \in \mathbb{N} \backslash\{0\}$, we define $\psi_{n}:=\varphi_{n} \chi_{1}$, where, for example,

$$
\varphi_{n}(s):=\left\{\begin{array}{lll}
1 & \text { if } & |s| \in[0, n), \\
(2 n-|s|) / n & \text { if } & |s| \in[n, 2 n), \\
0 & \text { if } & |s| \in[2 n, \infty) .
\end{array}\right.
$$

Although $\psi_{n}$ is not smooth, it is continuous and as such it as an admissible trial function from $\operatorname{Dom} Q$. Since the variables $(s, u)$ are separated in $\psi_{n}$, we arrive easily at

$$
\tilde{Q}\left[\psi_{n}\right]=\left(\psi_{n, s}, f^{-1} \psi_{n, s}\right)-\frac{1}{2}\left(\psi_{n}, K f \psi_{n}\right),
$$

where the first term vanishes as $n \rightarrow \infty$ because

$$
0<\left(\psi_{n, s}, f^{-1} \psi_{n, s}\right) \leq c_{-}^{-1}\left\|\varphi_{n}^{\prime}\right\|_{L^{2}(\mathbb{R})}^{2}=\frac{2 c_{-}^{-1}}{n} .
$$


We have employed here $\langle\mathrm{H} 2\rangle$ and the normalisation of $\chi_{1}$. Since $\varphi_{n} \rightarrow 1$ pointwise as $n \rightarrow \infty$ and $K$ is supposed to be integrable, the second term in (19) converges to the negative integral of (17) by the dominated convergence theorem. Consequently, there exists a fixed $n_{0}$ such that $\tilde{Q}\left[\psi_{n_{0}}\right]$ is negative and the proof is finished.

It may not be easy to verify the sufficient condition (18) for a given ambient surface $\mathcal{A}$ and reference curve $\Sigma$. Nevertheless, it is clear that it holds true for any strip of positive curvature. On the other hand, the condition is not satisfied for the strips in the plane where, however, it is well known that any non-trivial curvature of $\Sigma$ pushes the spectrum of $H$ below the energy $\kappa_{1}^{2}$. The following result shows that the same holds true for a more general class of quantum strips, including the flat case too.

Theorem 3. Assume $\langle\mathrm{H} 1\rangle,\langle\mathrm{H} 2\rangle,\langle\mathrm{H} 4\rangle$, and suppose that

$$
\left(\chi_{1}, K \chi_{1}\right)_{\mathcal{H}}=0 \text {. }
$$

If $K \equiv 0$, we require in addition that $k \not \equiv 0$. Then

$$
\inf \sigma(H)<\kappa_{1}^{2} .
$$

Proof: Let us start with formal considerations. By virtue of (17), the condition (20) implies that $\tilde{Q}\left[\chi_{1}\right]=0$. It is the result which one obtains for the strips in the plane. There the usual strategy is to deform slightly the function $\chi_{1}$ on a curved part of the strip in order to obtain a negative value of the functional $\tilde{Q}$. In particular, let $\varepsilon \in \mathbb{R}$ and there exist a function $\phi$ of a compact support in $\Omega_{0}$ such that it belongs to $\operatorname{Dom} Q$ and $\tilde{Q}\left(\phi, \chi_{1}\right)$ is not equal to zero. Writing

$$
\tilde{Q}\left[\chi_{1}+\varepsilon \phi\right]=\tilde{Q}\left[\chi_{1}\right]+2 \varepsilon \tilde{Q}\left(\phi, \chi_{1}\right)+\varepsilon^{2} \tilde{Q}[\phi],
$$

and since the first term at the r.h.s. equals zero, we can choose $\varepsilon$ sufficiently small and of a suitable sign so that the sum of the last two terms is negative. The result is then justified by using the mollifier $\varphi_{n}$ from the proof of the previous theorem in order to regularise $\chi_{1}$. Since the function $\varphi_{n}$ equals one on an interval growing as $n \rightarrow \infty$ and $\phi$ is of a compact support, we can take $n$ sufficiently large so that $\tilde{Q}\left(\phi, \varphi_{n} \chi_{1}\right)$ does not depend on $n$. Hence it suffices to find an appropriate function $\phi$ which verifies the above properties.

If $K \not \equiv 0$, we take $\phi(s, u):=j(s, u)^{2} \chi_{1}^{\prime}(u)$, where $j$ is a non-zero infinitely smooth function with a compact support on a region in $\Omega_{0}$ where $f_{, u}$ does not change sign and it is not identically zero. Such a region surely exists because $f_{, u}$ is a continuous function satisfying (5). Then

$$
\tilde{Q}\left(\phi, \chi_{1}\right)=-\left(j \chi_{1}^{\prime}, f_{, u} j \chi_{1}^{\prime}\right) \neq 0 .
$$

If $K \equiv 0$, we take $\phi(s, u):=j(s)^{2} u \chi_{1}(u)$, where $j$ is a non-zero infinitely smooth function with a compact support on an interval in $\mathbb{R}$ where $k$ does not change sign and it is not identically zero. Then

$$
\tilde{Q}\left(\phi, \chi_{1}\right)=\frac{1}{2}\left(j \chi_{1}, f_{, u} j \chi_{1}\right)=(j, k j)_{L^{2}(\mathbb{R})} \neq 0,
$$

where we have used the explicit form (7) of $f$ for the flat strips. 
Remark 6 . If $K \equiv 0$, we have already mentioned that the idea of the proof belongs to [14. Nevertheless, the deformation is not given explicitly there. An explicit deformation function is used in [6], however, we have not used it here because it would require an extra condition on the regularity of $f$. Our trial function is inspired with [25]; see also [8, Thm. 5.1].

An immediate consequence of Theorems 1, 28 and 3 is the following

Corollary 1. Assume $\langle\mathrm{H} 1\rangle,\langle\mathrm{H} 2\rangle,\langle\mathrm{H} 3\rangle,\langle\mathrm{H} 4\rangle$, and suppose that

$$
\left(\chi_{1}, K \chi_{1}\right)_{\mathcal{H}} \geq 0 \text {. }
$$

If $K \equiv 0$, we require in addition that $k \not \equiv 0$. Then

$$
\sigma_{\text {disc }}(H) \neq \emptyset \text {, }
$$

ie, there exists at least one isolated eigenvalue of finite multiplicity situated below $\kappa_{1}^{2}$.

\section{Non-Negative Curvature}

Since the condition (21) is clearly satisfied for non-negatively curved strips, we shall suppose that $K \geq 0$ through all this section and investigate this situation in detail. Since the integral $\left(\chi_{1}, K \chi_{1}\right)_{\mathcal{H}}$ is always well defined, we may not assume the assumption $\langle\mathrm{H} 4\rangle$. This includes to use the monotone convergence theorem instead of the dominated one we have used in the proofs of Theorems 2 and 3 .

An integration of the Jacobi equation (5) yields the following identity

$$
\forall(s, u) \in \Omega_{0}: \quad f_{, u}(s, u)=k(s)-\int_{0}^{u} K(s, \xi) f(s, \xi) d \xi .
$$

Since $K$ is non-negative, we have immediately

$$
f(s, u) \leq 1+u k(s) .
$$

Let $a\|k\|_{\infty}<1$. Putting the inequality (23) into (22), we get an opposite bound

$$
f(s, u) \geq 1+u k(s)-\frac{1}{2} u^{2}\left(1+\frac{1}{3} u k(s)\right) \sup _{\xi \in I} K(s, \xi) .
$$

It follows from (23) and (24) that the condition $\langle\mathrm{H} 2\rangle$ can always be achieved for bounded curvatures and $a$ small enough. More specifically, a condition on the half-width is expressed by means of the following inequality

$$
\frac{1}{6} a^{2}\|K\|_{\infty}+\frac{2}{3-a\|k\|_{\infty}}<1 .
$$

(The supremum norm of $K$ is taken over the strip only.) We note that the condition $a\|k\|_{\infty}<1$ is a usual assumption in the theory of quantum strips in the plane, while the presence of $K$ in (25) is due to the curved ambient space $\mathcal{A}$.

Furthermore, it is clear from (23) and (24) that the asymptotic condition $\langle\mathrm{H} 3\rangle$ is satisfied if we assume 


$$
k \stackrel{\infty}{\longrightarrow} 0 \quad \text { and } \quad K \stackrel{\infty}{\longrightarrow} 0 .
$$

We refer to the beginning of Section 1 for the exact definition of " $\underset{\longrightarrow}{\longrightarrow}$ ". The first limit is the usual assumption on the asymptotic straightness of the strips in the plane, while the second requires that the surface $\Omega$ is asymptotically flat. The latter restricts the asymptotic behaviour of the ambient space $\mathcal{A}$.

Finally, we remind that also the basic assumption $\langle\mathrm{H} 1\rangle$ can always be achieved for sufficiently small $a$ if one assumes in addition that the strip does not overlap, $c f$ Remark 2. Summing up the above considerations together with the results of the precedent sections, we conclude by

Theorem 4. Let $\Omega$ be a strip of non-negative curvature, $K \geq 0$, which does not overlap and satisfies the condition (25) together with a\|k$\|_{\infty}<1$. If it is not a geodesic strip, $k \neq \equiv$ or $K \not \equiv 0$, then $\inf \sigma(H)<\kappa_{1}^{2}$. If it is in addition an asymptotically geodesic strip, $\left\langle\mathrm{H} 3^{\prime}\right\rangle$, then the essential spectrum starts above $\kappa_{1}^{2}$ and $H$ has at least one isolated eigenvalue of finite multiplicity.

This theorem generalises the known results for strips in the plane, [6], which are a particular case of the flat strips, $K \equiv 0$. Moreover, the condition which enables us to localise the essential spectrum is weaker in the sense that it does not contain derivatives of the curvature $k$ of the reference curve. However, the most important generalisation concerns the quantum strips on non-trivially curved manifolds with a positive curvature. An instructive example in $\mathbb{R}^{3}$ is given by the infinite strips on the paraboloid of revolution.

\section{Ruled Strips}

In Section 2.4, we have found an explicit form of the metric (4) in Fermi coordinates for the flat strips which represent a trivial situation $(K \equiv 0)$. In general, however, it is not at all an easy problem to find $f$ because it requires to determine the geodesics orthogonal to the reference curve $\Sigma$ and integrate the Jacobi equation (5) over these geodesics. Nevertheless, there is a non-trivial class of strips in $\mathbb{R}^{3}$ where the metric is easy to calculate. For, consider the strip $\Omega$ constructed by segments orthogonal to a space curve $\Sigma$. Such a strip is a part of a ruled surface $\mathcal{A}$ based on $\Sigma$, 20, Def. 3.7.4]. As we have mentioned in the introduction, the Hamiltonian $H$ of a quantum particle in the ruled strips had already been investigated in [2]. The aim of the present paper is just to derive another expression for $f$, which suits better to our approach, and discuss some properties of $H$. A more detailed spectral analysis of the ruled strips will be discussed elsewhere.

Let $\Sigma$ be a simple, infinite curve of class $C^{3}$ in $\mathbb{R}^{3}$ and $p: \mathbb{R} \rightarrow \mathbb{R}^{3}$ be its parameterisation by the arc length $s$. We assume that the set $\{\dot{p}, n, b\}$, where $n$ and $b$ are the unit normal and binormal vectors, respectively, is well defined and forms a right-handed Frenet triad frame. We use the symbols $\kappa$ and $\tau$ for the curvature and torsion of $\Sigma$, respectively. One general class of ruled surfaces $\mathcal{A}$ 
is defined via $\mathcal{L}: \mathbb{R}^{2} \rightarrow \mathbb{R}^{3}$,

$$
\mathcal{L}(s, u):=p(s)+u[n(s) \cos \theta(s)-b(s) \sin \theta(s)],
$$

where $\theta: \mathbb{R} \rightarrow \mathbb{R}$ is a function of class $C^{1}$. The ruled strip $\Omega$ is then given by (3) so that $\langle\mathrm{H} 1\rangle$ and $\langle\mathrm{H} 2\rangle$ hold true. The mapping (26) does really represent the Fermi-coordinate chart (2) with the metric of the form (4). Employing the Frenet-Serret formulae, an explicit calculation yields

$$
\begin{aligned}
& f(\cdot, u)^{2}=(1-u \kappa \cos \theta)^{2}+u^{2}\left(\tau-\theta^{\prime}\right)^{2} \\
& K(\cdot, u)=-\frac{\left(\tau-\theta^{\prime}\right)^{2}}{f(\cdot, u)^{2}}, \quad k=-\kappa \cos \theta .
\end{aligned}
$$

It is clear that any ruled strip has always a non-positive curvature. Consequently, the sufficient condition (21) is achieved only in the limit case, $K \equiv 0$, which corresponds to $\theta^{\prime}=\tau$. In that case, $\Omega$ is a flat strip which may not be necessary a part of plane, however.

Combining (27) with (28), we get

$$
f(s, u)=\frac{1+u k(s)}{\sqrt{1+u^{2} K(s, u)}},
$$

which is an expression of a more transparent structure from the intrinsic point of view of this paper. At the same time, it is clear from (29) that the condition $\langle\mathrm{H} 2\rangle$ holds true provided

$$
a\|k\|_{\infty}<1 \quad \text { and } \quad a^{2}\|K\|_{\infty}<1,
$$

and the assumption $\langle\mathrm{H} 1\rangle$ then follows by the additional requirement that $\Omega$ does not overlap. Next, the ruled strip is asymptotically geodesic under the assumption $\left\langle\mathrm{H} 3^{\prime}\right\rangle$, which implies that inf $\sigma_{\mathrm{ess}}(H) \geq \kappa_{1}^{2}$ by Theorem 1 . However, an open question is whether there exist bound states below the threshold of the essential spectrum provided $K \not \equiv 0$.

\section{Concluding Remarks}

The main interest of this paper was to investigate spectral properties of the Laplacian $-\Delta$, subject to Dirichlet boundary conditions, in the strip region $\Omega$ defined as the tubular neighbourhood of an infinite curve $\Sigma$ in a two-dimensional Riemannian manifold $\mathcal{A}$. The strategy was to express the operator $-\Delta$ under suitable assumptions, $\langle\mathrm{H} 1\rangle,\langle\mathrm{H} 2\rangle$, in geodesic coordinates based on $\Sigma$. We were mainly interested in the existence of the discrete spectrum. In particular, using some variational techniques, we proved that there are bound states below the essential spectrum provided the strip is not geodesic, $K \not \equiv 0$ or $k \not \equiv 0$, but asymptotically geodesic, $\langle\mathrm{H} 3\rangle$, and positively curved "in the mean" in the sense of (21). The latter sufficient conditions hold particularly true for the strips of a 
non-negative curvature which were investigated in detail. The obtained results represent a generalisation of quantum strips in the plane, [6].

An interesting problem is to decide whether the discrete spectrum exists for some negatively curved quantum strips as well. The simplest model is probably given by the ruled strips introduced in the previous section. It is also desirable to investigate the spectrum of quantum strips on surfaces more precisely using some perturbation and numerical methods. Another direction of a future research consists in quantum strips which are not asymptotically geodesic; this may include periodically or randomly curved strips too. Following [5], we also expect that interesting new features may be brought by a switch of the boundary condition. Apart from the spectral analysis, the scattering problem represents another challenge facing the theory of quantum strips.

The present paper has been motivated by the theory of quantum waveguides. If one deals with a curved quantum waveguide in the plane, a reasonable model is given by the two-dimensional Laplacian in an infinite strip in $\mathbb{R}^{2}$, [6]. However, we stress here that the two-dimensional Laplacian in the strip on a curved surface does not represent the actual Hamiltonian of a space quantum waveguide. For, a quantum particle in a strip-like waveguide is forced to move close to $\Omega$ by means of a constraining potential (representing a high chemical potential between different semiconductor materials) but, due to tunneling effect, it can be found, even if not too far, outside the strip in the space $\mathbb{R}^{3}$ too. Even if this effect is not important for the waveguide in the plane because the motion of the particle in the direction transverse to the plane can be separated, it is not negligible for waveguides on a curved surface. In this paper, we dealt with a more general situation when the ambient space $\mathcal{A}$ of the waveguide may not be embedded in $\mathbb{R}^{3}$. Our results are interesting from the mathematical point of view, however, it is worth to know whether they could be interpreted physically as well.

\section{Acknowledgments}

The author would like to thank Professors Pierre Duclos, Pavel Exner, and Jean Nourrigat for useful discussions. The work has been partially supported by GA AS CR grant IAA 1048101.

\section{References}

[1] I. J. Clark and A. J. Bracken, Bound states in tubular quantum waveguides with torsion, J. Phys. A 29 (1996), 4527-4535.

[2] __ Effective potentials of quantum strip waveguides and their dependence upon torsion, J. Phys. A 29 (1996), 339-348.

[3] R. C. T. da Costa, Quantum mechanics of a constrained particle, Phys. Rev. A 23 (1981), 1982-1987. 
[4] E. B. Davies, Spectral theory and differential operators, Camb. Univ Press, Cambridge, 1995.

[5] J. Dittrich and J. Kř́ž, Curved planar quantum wires with Dirichlet and Neumann boundary conditions, to appear in J. Phys. A. E-preprint: mp_arc 02-100 or math-ph/0203007.

[6] P. Duclos and P. Exner, Curvature-induced bound states in quantum waveguides in two and three dimensions, Rev. Math. Phys. 7 (1995), 73-102.

[7] P. Duclos, P. Exner, and D. Krejčiřík, Locally curved quantum layers, Ukrainian J. Phys. 45 (2000), 595-601.

[8] _ Bound states in curved quantum layers, Commun. Math. Phys. 223 (2001), 13-28.

[9] P. Exner and D. Krejčiř́ík, Bound states in mildly curved layers, J. Phys. A 34 (2001), 5969-5985.

[10] P. Exner and P. Šeba, Bound states in curved quantum waveguides, J. Math. Phys. 30 (1989), 2574-2580.

[11] E. Fermi, Sopra i fenomeni che avvengono in vicinanza di una linea oraria, Atti R. Accad. Lincei Rend. Cl. Sci. Fis. Mat. Natur. 31 (1922), 21-23, 51-52, 101-103. Also The Collected Works of E. Fermi 1, The University of Chicago Press, Chicago (1962), 17-19.

[12] F. Fiala, Le problème des isopérimètres sur les surfaces ouvertes à courbure positive, Comment. Math. Helv. 13 (1940-41), 293-346.

[13] R. Froese and I. Herbst, Realizing holonomic constraints in classical and quantum mechanics, Commun. Math. Phys. 220 (2001), 489-535.

[14] J. Goldstone and R. L. Jaffe, Bound states in twisting tubes, Phys. Rev. B 45 (1992), 14100-14107.

[15] A. Gray, Tubes, Addison-Wesley Publishing Company, New York, 1990.

[16] P. Hartman, On the local uniqueness of geodesics, Amer. J. Math. 72 (1950), 723-730.

[17] _ Geodesic parallel coordinates in the large, Amer. J. Math. 86 (1964), 705-727.

[18] P. Hartman and A. Wintner, On the third fundamental form of a surface, Amer. J. Math. 75 (1953), 298-334.

[19] H. Jensen and H. Koppe, Quantum mechanics with constraints, Ann. Phys. 63 (1971), 586-591.

[20] W. Klingenberg, A course in differential geometry, Springer-Verlag, New York, 1978. 
[21] H. Koppe and H. Jensen, Das prinzip von d'Alembert in der klassischen mechanik und in der quantentheorie, Sitzungber. der Heidelberger Akad. der Wiss., Math.-Naturwiss. Klasse 5 (1971), 127-140.

[22] J. T. Londergan, J. P. Carini, and D. P. Murdock, Binding and scattering in two-dimensional systems, LNP, vol. m60, Springer, Berlin, 1999.

[23] K. A. Mitchell, Gauge fields and extrapotentials in constrained quantum systems, Phys. Rev. A 63 (2001), art. 042112.

[24] M. Reed and B. Simon, Methods of modern mathematical physics, vol. IV Analysis of Operators, Academic Press, New York, 1978.

[25] W. Renger and W. Bulla, Existence of bound states in quantum waveguides under weak conditions, Lett. Math. Phys. 35 (1995), 1-12.

[26] J. Tolar, On a quantum mechanical d'Alembert principle, Group theoretical methods in physics, LNP, vol. 313, Springer, 1988, pp. 268-274. 